\title{
Immediate spatial distortions of pointing movements induced by visual landmarks
}

\author{
JÖRN DIEDRICHSEN \\ University of California, Berkeley, California \\ STEFFEN WERNER \\ University of Idaho, Moscow, Idaho \\ THOMAS SCHMIDT \\ Georg-August-Universität, Göttingen, Germany \\ and \\ JULIA TROMMERSHÄUSER \\ New York University, New York, New York
}

\begin{abstract}
We tested the influence of two horizontally aligned visual landmarks on pointing movements to memorized targets, to investigate whether the visuomotor system can make use of an egocentric representation unaffected by visual context. The endpoints of pointing movements were systematically distorted toward the nearest visual landmark, indicating that spatial representations included both target and nontarget information. These distortions were not due to the presence of the landmarks during the movement but, rather, to their presence in the encoding phase. Qualitatively similar distortions were present even with the shortest possible retention phase, when the target was extinguished at movement onset. Finally, we found the same pattern of distortion when participants were forced to remember the target within an allocentric frame of reference. We argue that even early memory representations for pointing movements are influenced by visual information in the surrounding visual field.
\end{abstract}

It has been suggested that humans encode the spatial locations of objects in the environment by using a multitude of spatial representations, so-called maps. To guide eye and arm movements toward visible objects, a cascade of coordinate transformations is necessary to translate the target location from retinotopic coordinates to a viewer-centered coordinate system that can be used to execute the movement (Buneo, Jarvis, Batista, \& Andersen, 2002; Flanders, Tillery, \& Soechting, 1992; McIntyre, Stratta, Droulez, \& Lacquaniti, 2000). These spatial maps represent the location of the target in relation to the observer (egocentric information). However, we are also clearly capable of encoding and remembering the spatial relationships among different objects, invariant to the position of our body (allocentric information).

This research was supported by Grant DFG-We 1973/3 from the German science foundation to S.W. We thank Björn Rump and Christopher Hirsch for assistance with data collection and Dan Simons, Dirk Kerzel, Joseph McIntyre, and William Prinzmetal for helpful comments on an earlier version of the manuscript. Correspondence concerning this article should be addressed to J. Diedrichsen, Department of Biomedical Engineering, Johns Hopkins University, 720 Rutland Ave., 419 Traylor Building, Baltimore, MD 21205-2195(e-mail: jdiedric@bme.jhu.edu).

Note-This article was accepted by the previous editorial team, headed by Neil Macmillan
Egocentric and allocentric representations have been associated with the dorsal and the ventral processing streams arising from early visual areas and terminating in the posterior parietal cortex and the inferior temporal cortex, respectively (e.g., Merigan \& Maunsell, 1993). In humans, a dissociation between these two visual systems has primarily been shown in brain-damaged patients: Lesions to the dorsal stream can result in visuomotor impairment despite intact object recognition (Perenin \& Vighetto, 1988), whereas lesions to the ventral stream might lead to dense object agnosia despite largely intact visuomotor functions (Goodale, Milner, Jakobson, \& Carey, 1991; Milner et al., 1991).

Behavioral studies in neurologically unimpaired persons have suggested that direct motor actions rely mostly on egocentric information, whereas perception and cognitive judgments rely to a larger degree on allocentric information. These studies typically show that the perception of an object is affected by the surrounding visual context, whereas a motor action in an analogous situation is not affected by it or is affected to a lesser degree.

For example, in a study by Bridgeman, Peery, and Anand (1997; see also Bridgeman, Gemmer, Forsman, \& Huemer, 2000), participants judged the spatial position of a target dot within a rectangular frame. When the frame was displayed to the left or right of the body midline, perceptual judgments of target position were biased in the di- 
rection opposite to the displacement of the frame. However, this bias was not present in direct pointing responses toward the target. The crucial assumption behind this and other comparable studies is that the targeted spatial endpoint is represented relative to the body of the observer in such a way that this representation is independent of the surrounding visual context. Throughout this paper, we will refer to a representation with this property as a context-independent egocentric representation.

In the contrasting case, a spatial representation might include multiple objects and locations and the relationships among them. In this situation, interactions between the different parts might lead to specific illusions or distortions that critically depend on the layout of the visual display. It is important to note that context-dependentspatial distortions do not necessarily imply that the map in which these distortions arise is coded with respect to an allocentric reference frame. If multiple objects are encoded with respect to an egocentric reference frame and if these representations are allowed to interact, context dependency can arise in an egocentric map as well. However, such a representation would include not only information about the positions relative to the body of the observer, but also information about the interrelations of the objects-that is, allocentric information. Context-independent egocentric representations and allocentric spatial representations that are independent of the location of the observer are the extreme points of a continuum on which spatial representations can lie (Pouget \& Sejnowski, 1997).

In investigating spatial responses, two aspects are typically considered. The constant error refers to the difference between the mean reproduced location and the actual target location. Any distortions in the participant's representation of the location would manifest itself in this measure. The variable error indicates the spread of the individual responses around the mean reproduced location and can be visualized as confidence ellipses. The orientation of these error ellipses often allows inferences about the structure of the underlying representation.

For example, in a study by Rossetti and co-workers (Rossetti, 1998), participants were required to point accurately to the target location when a go signal was provided within $0-8 \mathrm{sec}$ following target presentation. For immediate responses, the confidence ellipses indicating the variable error of movement endpoints were aligned with movement direction, suggesting that mostly egocentric information was used to guide the movement. For delays longer than $1 \mathrm{sec}$, the distribution of endpoints depended on visual context, indicating the use of allocentric information. This study suggests that the influence of the visual context on pointing or eye movements critically depends on the delay between stimulus representation and the initiation of the action.

Similarly, Carrozzo, Stratta, McIntyre, and Lacquaniti (2002) showed that if all possible target points were arranged on a line, the confidence ellipses of the movement endpoints tended to be aligned with this constructed allocentric reference frame, even though the line was never explicitly shown to the participants. Again, this influence of an allocentric reference was much more pronounced after a memory interval of $5 \mathrm{sec}$ than after $0.5 \mathrm{sec}$.

A similar dependence on the retention interval was also found for pointing movements toward an apex of a MüllerLyer stimulus (Gentilucci, Chieffi, Daprati, Saetti, \& Toni, 1996). Movements that were initiated immediately after visual feedback was withdrawn were only slightly influenced by the illusion, whereas the movements initiated $5 \mathrm{sec}$ later showed a substantial influence of the illusion (see also Elliott \& Lee, 1995; Hu \& Goodale, 2000).

From these studies, it seems that the motor system makes use of allocentric spatial information to guide hand movements toward memorized targets. However, it is still a matter of controversy whether immediate action may be guided by a context-independentegocentric representation and, if so, how long such a representation might persist. Several authors have argued that there might be a specialized motor short-term memory capable of storing egocentric information independently of a general visual short-term memory store (Bridgeman, Lewis, Heit, \& Nagle, 1979). This store could underlie the ability to remember the endpoint of a recently executed reaching movement (Kelso, 1977; Laabs, 1973; Stelmach, Kelso, \& Wallace, 1975), to execute a delayed saccade (Gnadt \& Andersen, 1992), or to reach toward an object without visual guidance (Pelisson, Prablanc, Goodale, \& Jeannerod, 1986). Sustained neural activity during delayed reaching tasks has been observed in dorsal stream areas (Smyrnis, Taira, Ashe, \& Georgopoulos, 1992) and also in the prefrontal cortex (Funahashi, Bruce, \& Goldman-Rakic, 1989; Goldman-Rakic, 1995), constituting possible neural correlates of such a motor shortterm memory system.

Visual context can lead to systematic distortions when participants are asked to reproduce the position of a briefly presented target by using paper and pencil or a mouse cursor (Huttenlocher, Hedges, \& Duncan, 1991; Nelson \& Chaiklin, 1980). In our own work on the reproduction of dot location with a mouse cursor, we have demonstrated that reliable distortions are introduced by two horizontally aligned landmark dots (Diedrichsen, 1998; Schmidt, Werner, \& Diedrichsen, 2003; Werner \& Diedrichsen, 2002; Werner \& Schmidt, 2000). Targets presented near a landmark are remembered too far away from it, and there is an additional distortion away from the midpoint between the landmarks. At the same time, variable error of reproduced positions is greatly reduced near the landmarks. The dependence of the distortions on the layout of the landmarks is well established, because the distortion patterns shift, rotate, expand, and contract along with the landmarks (Werner \& Schmidt, 2000). Furthermore, distortions arise as early as $50 \mathrm{msec}$ following offset of the target, increase with longer retention intervals, and reach an asymptote at about $400 \mathrm{msec}$ poststimulus (Werner \& Diedrichsen, 2002).

Thus, spatial distortions ${ }^{1}$ induced by visual landmarks can provide a novel approach by which to investigate the characteristics of the spatial representation underlying point- 


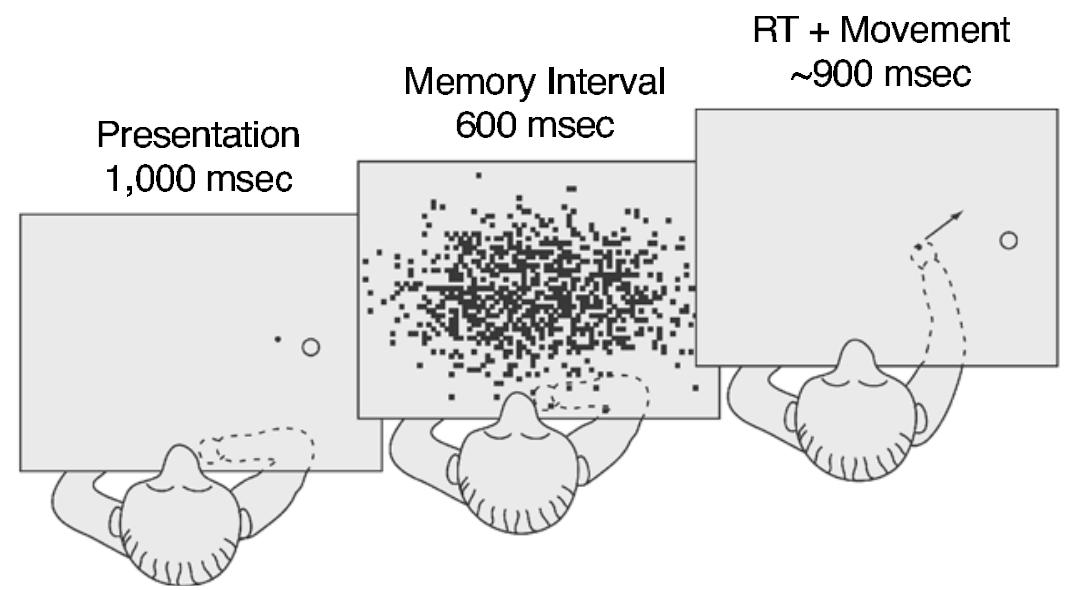

Figure 1. General procedure in the experiments. The participants sat in front of a table surface, their hands in a predefined starting area. In the presentation phase, a target dot and possible landmarks were presented. In this example, only one landmark is presented on the right side (unfilled circle). During the memory interval, the display was masked by a random and rapidly changing checkerboard pattern. The end of the mask and the reappearance of the landmark served as a starting signal for the movement phase. The participants were instructed to make a smooth pointing movement toward the memorized target location.

ing movements toward remembered targets. In a recent article, distortion patterns similar to those found by Huttenlocher et al. (1991) were reported for pointing movements (Gourtzelidis, Smyrnis, Evdokimidis, \& Balogh, 2001). These distortions were present when participants pointed to the targets after a 6-sec delay period and even, although they were somewhat weaker, when participants pointed to the targets immediately after the offset of the target. However, in that study, movements were made on a graphic tablet on a table, whereas the targets and visual movement feedback were presented on a vertically aligned monitor. A scaling factor of 0.7 was introduced between the spatial distances of the movements and on the visual display. This visuomotor transformation may have rendered the task more akin to the manipulation of a mouse cursor than to natural pointing movements and may have biased participants toward the use of allocentric information.

In this study, we investigated the question of whether natural pointing movements toward remembered targets rely on an undistorted egocentric representation by studying the influence of visual landmarks. Because such distortions develop very rapidly in the context of a perceptual judgment task (Werner \& Diedrichsen, 2002), they can be used to differentiate a purely egocentric representation from one that is influenced by allocentric information even for very short memory delays. Experiment 1 showed that memory representations are systematically distorted by visual landmarks even when pointing, rather than cursor movements, are used and that these distortions are organized within a context-dependent frame of reference. Experiment 2 showed that distortions are due to the presence of landmarks in the encoding phase and not to their sudden onset during the movement. In Experiment 3, we in- vestigated the development of the distortion with increasing retention intervals up to $600 \mathrm{msec}$. We showed that these effects were present even when the target was extinguished no earlier than at the onset of the pointing movement. Finally, in Experiment 4 we demonstrated that the distortions were qualitatively similar when the stimulus configuration was shifted with respect to the observer and participants were forced to reproduce the target position relative to the landmarks. Taken together, the results indicate that pointing movements are based on context-dependent spatial representations that include allocentric landmark-to-target information.

\section{EXPERIMENT 1}

Experiment 1 was designed to investigate whether pointing movements to memorized targets are susceptible to context-dependent distortions. The participants were instructed to remember the exact position of a target dot that was briefly presented to them on a table surface. After a brief interval in which a visual mask was presented, they had to point to the remembered location of the dot (Figure 1). In addition, either one or two landmarks were presented during the presentation and reproduction intervals. Note that the landmarks were irrelevant to the task but could be used as a spatial reference.

We expected that if the target was represented in a context-dependent map including allocentric relations among multiple objects, we would observe a systematic pattern of spatial distortions throughout the visual field that would resemble the one found with cursor movements (Schmidt et al., 2003; Werner \& Diedrichsen, 2002). Importantly, distortion effects should strongly depend on 
whether one or two landmarks were presented. In contrast, any pattern of bias arising within a context-independent reference system would be expected to be independent of the spatial layout of the landmarks.

\section{Method}

Participants. Nine undergraduate students from the University of California, Berkeley, participated in the experiment. Their age ranged from 18 to 20 years. Participation in the two 1-h sessions partly fulfilled course requirements.

Apparatus and Stimuli. The participants were seated at a $75-\mathrm{cm}-$ high table surface. An LCD projector (Plus UP 800) controlled by an IBM-compatible $486 \mathrm{PC}$ was used to project stimuli from above onto a rectangular cotton screen $(100 \times 77 \mathrm{~cm})$, which was mounted $48 \mathrm{~cm}$ above the table surface. The participants positioned their heads in a chinrest so that eye level was approximately $43 \mathrm{~cm}$ above the table. They viewed the screen from below through a mirror that was mounted halfway between and parallel to the table surface and the screen. With this setup, stimuli projected onto the screen appeared to lie on the table surface. The participants made the pointing movements below the mirror so that they could not see the limb. A magnetic tracking device (mini-bird system, Ascension Technologies) with a sampling rate of $130 \mathrm{~Hz}$ and a spatial resolution of around $0.5 \mathrm{~mm}$ was used to record the movements. For this purpose, a small $(1.7 \times 0.8 \times 0.8 \mathrm{~cm})$ receiver antenna was attached to the tip of the index finger of the right hand. A small dot $(2 \mathrm{~mm}$ diameter) was presented at the location corresponding to the position of the index finger in order to give the participants visual feedback about the position of the hand.

The target dot was white and had a diameter of $2 \mathrm{~mm}$. Unfilled circles with a diameter of $1.3 \mathrm{~cm}$ were used as landmarks. They were presented $12 \mathrm{~cm}$ to the left or right of the midline of the participant's body and $38 \mathrm{~cm}$ inward from the edge of the table surface. We used an array of 37 possible target locations between and around the possible landmark positions, arranged in a rectangular $15 \times 5$ grid. About half of the positions remained untested (the origins of the vectors in Figure 2 indicate the tested positions).

Procedure. A trial started when the participant moved the right index finger into the starting area and left it there for $800 \mathrm{msec}$. This area was defined as a $10 \times 20 \mathrm{~cm}$ rectangle along the lower edge of the table surface, $28 \mathrm{~cm}$ below the landmarks. A trial began with a 1,000 -msec presentation of the target dot at one of the 37 possible locations, together with the left, the right, neither, or both landmarks.

Presentation of target and landmarks was followed by a highintensity flicker mask. Great care was taken to use a mask that would effectively eliminate the visual trace of the target in sensory memory but could not be used as a spatial reference itself. A random checkerboard pattern of small squares $(3 \times 3 \mathrm{~mm})$ flickered at $60 \mathrm{~Hz}$ in an oval region covering the whole display for $600 \mathrm{msec}$. At the borders of the oval, the probability of white squares decreased in a Gaussian manner with increasing distance from the center, causing a smooth fading of the mask at the edges. The participants were not allowed to move their hands before the presentation of the mask was completed.

After the masking interval, the landmarks, if any, were presented again. Simultaneously, a dot appeared at the position of the hand. The disappearance of the mask was the starting signal to execute a smooth pointing movement to the remembered location. If the participants moved before the starting signal, the trial was aborted. The participants were encouraged to keep movement time between 700 and $800 \mathrm{msec}$ while being as accurate as possible. A brief beep was played as feedback after their movement had ended. The participants then had to move their hands back to the starting position to initiate the next trial.

All combinations of 37 possible target locations and four landmark conditions were presented once per block in pseudorandom order. After each block, feedback was given about the reaction time
(RT; measured from mask offset to movement onset), the movement time (MT; from movement onset to movement completion), and the average accuracy in centimeters. Over the course of two 1-h sessions, the participants received eight blocks of 148 trials, providing eight measurements per factor combination and participant. At the beginning of the first session, an additional practice block of 5 trials was given to acquaint the participants with the apparatus.

Data analysis. The movement data were smoothed with a Gaussian kernel of 6-msec width at half height. The onset and the end of the movement were computed as the time when the tangential velocity of the index finger first exceeded or fell below the threshold of $2.8 \mathrm{~cm} / \mathrm{sec}$ for at least $250 \mathrm{msec}$. The endpoint of the movement was defined as the average position over the 250 -msec rest period completing the movement.

For each participant and each target location separately, the constant error and variable error were computed. The constant error is the vector between the mean endpoint of the pointing movements and the target position. For statistical analyses, we treated the constant errors in horizontal $(x)$ and vertical $(y)$ directions separately, each pooled over the values of the other coordinate. We introduced this separation to reduce the degrees of freedom of the numerator, in order to make the tests stricter, without intending to imply that the two dimensions are indeed independent. Repeated measures analyses of variance (ANOVAs) were conducted with Greenhouse-Geisser corrected degrees of freedom and $p$ values. To determine whether there was a systematic pattern of distortion across participants, we tested whether the constant error differed between target locations, whereas differences between distortion patterns were tested by investigating the interaction between target location and experimental condition. A global shift across all target locations (the intercept term) was ignored by this analysis.

The variable error is the distance between each endpoint and the corresponding mean endpoint for that target location and subject. Using principle-component analysis, we computed the orientation of the axis of maximal and minimal variability and their eigenvalues for each participant and target position separately. The square root of the eigenvalues corresponds to the standard deviation of the movement endpoints along that axis.

\section{Results and Discussion}

All the trials in which no stable endpoint was reachedfor example, when there was a slow sliding motion of the hand-were excluded from analysis $(0.18 \%)$. Furthermore, all the trials in which the movement ended more than $5 \mathrm{~cm}$ distant from the presented target were also excluded (3.7\%). The average RT was $183 \mathrm{msec}$; the average MT was $753 \mathrm{msec}$.

The pattern of constant error (see Figure 2) provides evidence that the representation of the target location was influenced by the presence of the landmarks. A two-way repeated measures ANOVA (target position $\times$ landmark condition) confirmed that the pattern of distortions depended strongly on landmark condition, as was shown by significant interaction effects [for $x$-direction, $F(4.2,33.5)=$ $3.99, p=.009$; for $y$-direction, $F(3.4,27.1)=3.8, p=.018]$. Subsequent tests confirmed that distortion effects in the $x$ direction were present in the left-landmark, right-landmark, and both-landmarks conditions (all $F \mathrm{~s}>6.90$, all $p$ s $<$ $.003)$, but not in the no-landmark condition $[F(2.2,17.9)=$ $1.41, p=.272]$.

The systematic pattern of constant error in the twolandmarks condition (Figure 2, bottom panel) provides evidence that the positional information is context sensi- 

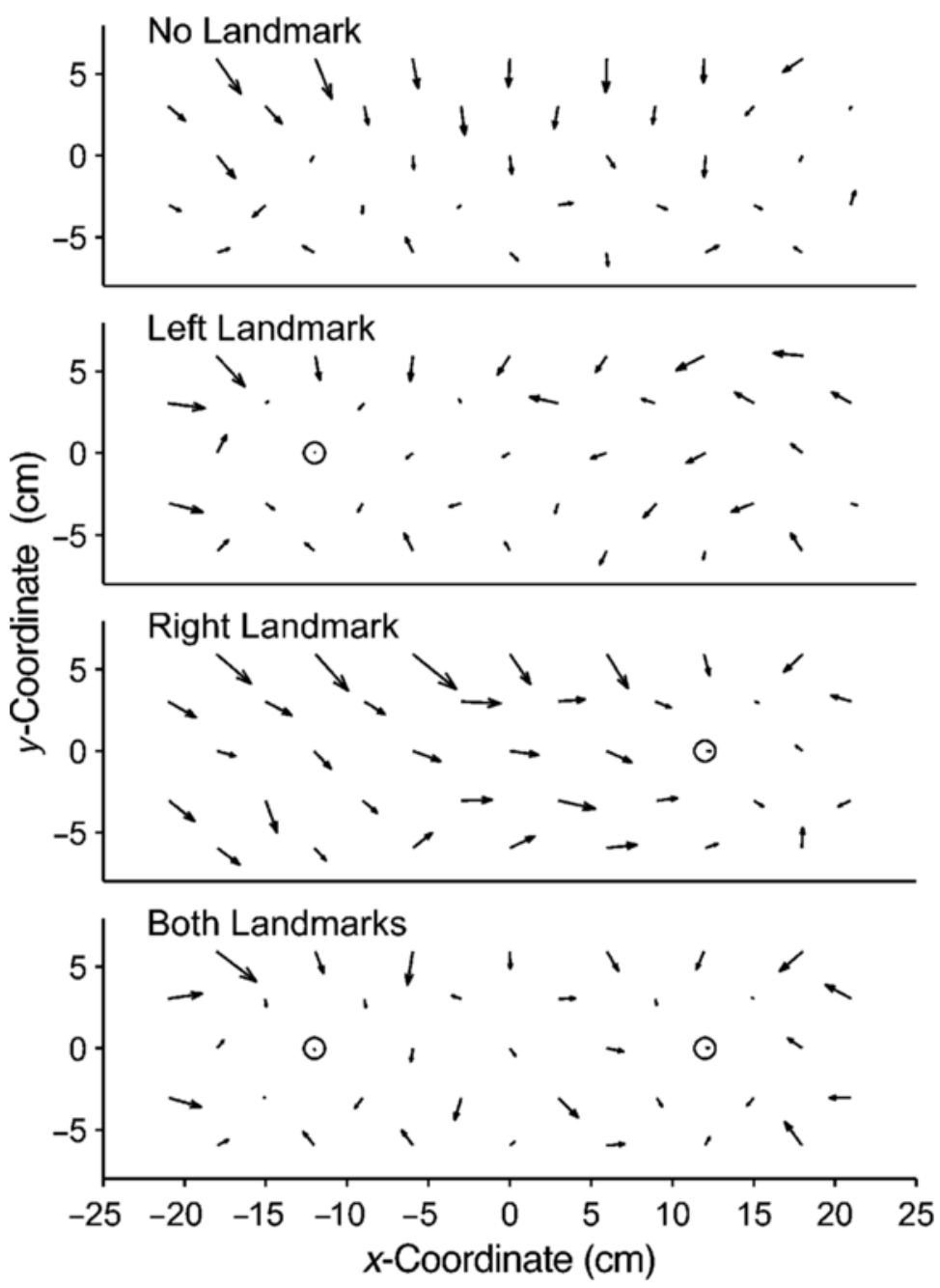

Figure 2. Vector plot of the average distance between the endpoint of the movement and the presented target location, indicating a constant error toward the nearest landmark. Results are shown for the no-landmark, left-landmark, right-landmark, and both-landmarks conditions. For better readability, the length of the vectors is magnified by a factor of three.

tive, indicating the relevance of allocentric landmark-toobject spatial relations. In contrast to the no-landmark condition, in which there was no systematic bias in the horizontal direction, remembered target positions were systematically distorted away from the midpoint and toward each landmark. This distortion followed the pattern of the left-landmark condition for the left side and of the right-landmark condition for the right side, suggesting that the distortion pattern was locally defined by the closest landmark (Schmidt et al., 2003). Regarding error in the $y$-direction, we found in the no-landmark condition a general tendency to undershoot far targets more $[F(1.1,9.1)=$ $4.84, p=.052]$. This tendency was strongly reduced in the presence of landmarks, where movements to targets on the imaginary line connecting the landmarks were accurate in terms of their amplitude. Overall, landmarks introduced a systematic pattern of spatial distortions that critically depended on whether the landmark was presented on the left, the right, or both sides of the display, suggesting that the target position was stored in a map that was spatially organized by nontarget visual stimuli.

If landmarks are indeed an integral part of the target representation, we would also expect that the variable error would be influenced by the landmarks, as has been observed in reproduction experiments (Schmidt et al., 2003; Werner \& Diedrichsen, 2002). In the no-landmark condition, the axis of maximal variability tended to align with the movement direction (Figure 3), as has been observed in previous studies (Carrozzo et al., 2002). With the introduction of landmarks, the variable error near the landmarks was reduced considerably, and its structure changed. To compare variable error when the landmark was or was 

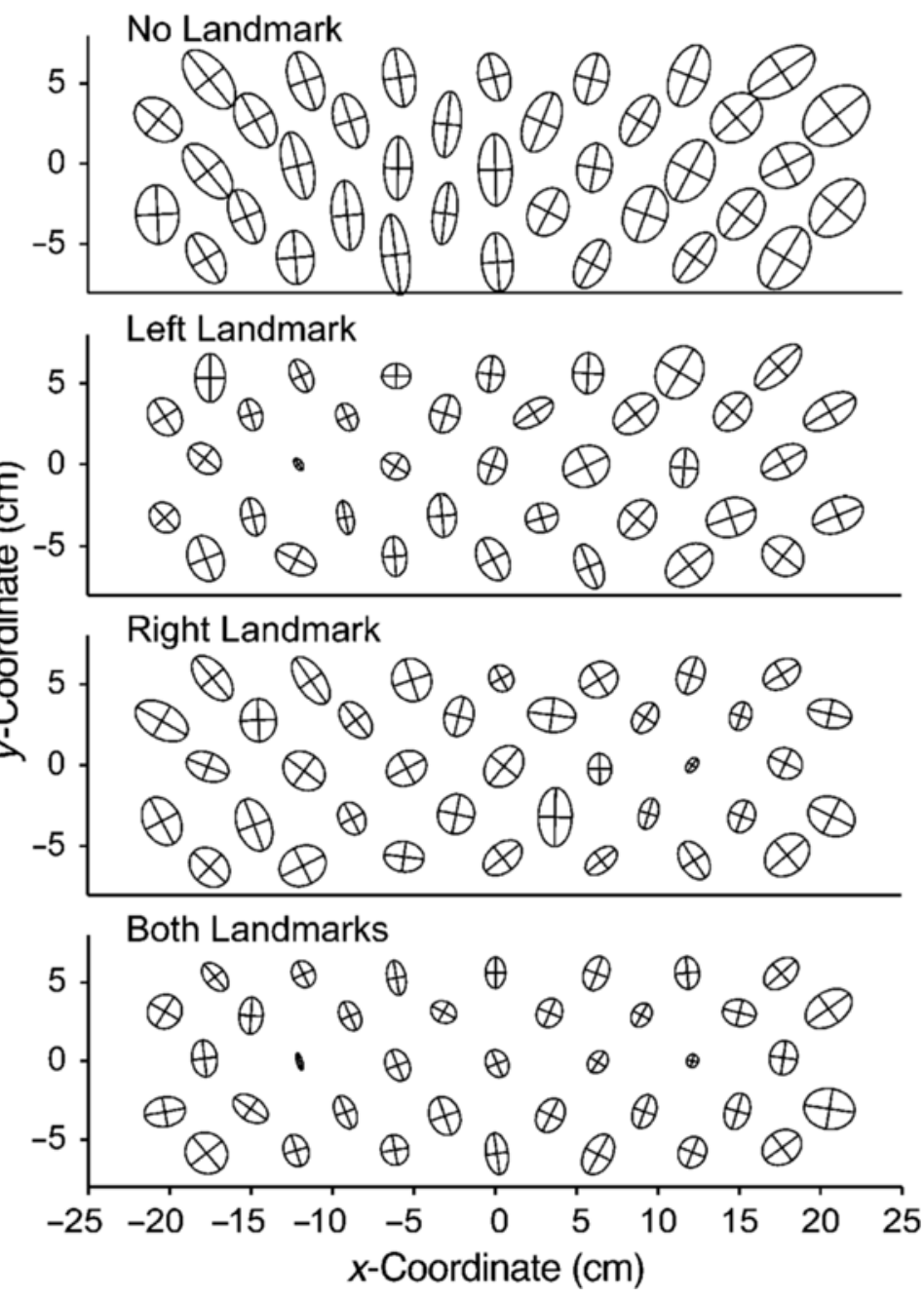

Figure 3. Variable error of movement endpoints in Experiment 1. To obtain a graphical representation of the average within-subjects (variable) error for each target point, we subtracted the participants' average reproduced location from the movement endpoints and pooled the residuals across participants. The ellipses indicate $1 S D$ dispersion, and the two orthogonal axes indicate the maximal and minimal variability of these residuals, centered around the average reproduced location for each target point.

not presented, we considered the data from the six positions immediately surrounding the possible landmark locations for the left and right halves of the display. Planned tests showed that variable error in the direction of maximal variability was substantially lower when a landmark was present than when it was absent $[F(1,8)=26.69, p<.001]$. The pattern of variable error suggests that landmarks served to reduce positional uncertainty in their vicinity.

Distortions away from the midpoint between the landmarks, as well as reductions of variability near the landmarks, are consistent with previous findings (Schmidt et al., 2003; Werner \& Diedrichsen, 2002). Strikingly, we did not find a distortion away from the landmarks themselves, which had been a typical finding in each of our previous experiments with mouse cursor instead of pointing movements. We will return to this issue in the General Discussion section.

\section{EXPERIMENT 2}

In the previous experiment, we attributed the pattern of distortion introduced by landmarks to the use of allocentric information in the guidance of pointing movements. However, there remained an alternative account for such an effect. After the memory interval and visual mask, the participants experienced the sudden visual onset of the landmarks. This onset likely captured covert visual attention (Yantis \& Jonides, 1990) and may have elicited an eye movement toward these landmarks. The tight coupling of 
Table 1

Reaction Times (RTs), Movement Times (MTs), and Variable Endpoint Errors (VEs) Along the Axes of Maximal and Minimal Variance in the Three Conditions of Experiment 2

\begin{tabular}{|c|c|c|c|c|c|c|}
\hline \multirow[b]{3}{*}{ Variable } & \multicolumn{6}{|c|}{ Experimental Condition } \\
\hline & \multicolumn{2}{|c|}{ Presentation Only } & \multicolumn{2}{|c|}{ Movement Only } & \multicolumn{2}{|c|}{ Both Phases } \\
\hline & Near & Far & Near & Far & Near & Far \\
\hline $\mathrm{RT}(\mathrm{msec})$ & 233 & 236 & 220 & 220 & 215 & 218 \\
\hline MT (mse & 728 & 789 & 721 & 775 & 784 & 804 \\
\hline VE maximal (cr & 0.73 & 0.74 & 0.70 & 0.71 & 0.53 & 0.60 \\
\hline VE minimal $(\mathrm{cm})$ & 0.32 & 0.34 & 0.31 & 0.31 & 0.25 & 0.28 \\
\hline
\end{tabular}

Note-Results are shown separately for the six locations immediately surrounding each landmark (near) and for the remaining locations (far).

hand and eye movements (e.g., Neggers \& Bekkering, 2000) might have led to an attraction of the hand path toward the landmark. ${ }^{2}$

In Experiment 2, we used a stimulus display with two landmarks in three variations. The landmarks either were visible during the presentation of the target, but not during the reproduction (presentation-only condition), or were visible only during the reproduction phase, but not during the presentation phase (movement-only condition). We also presented the landmarks during both presentation and reproduction, as in the previous experiment (both-phases condition).

If the distortion found in Experiment 1 was due to the sudden visual onset of the landmark stimuli immediately before the movement, the distortion should be present in the movement-only and both-phases conditions, but not in the presentation-only condition. If allocentric information is used strategically by participants to encode target positions relative to the nearest landmark and later to reproduce the location on the basis of this information, we should find distortions only for the both-phases condition. Finally, if the spatial relations to neighboring objects and landmarks are integrated in obligatory fashion into the representation used to guide movements, it should lead to distortions regardless of the presence of the visual context during the actual movement. If so, the characteristic attraction toward the landmark locations might be present in both the presentationonly and the both-phases conditions.

\section{Method}

Participants. Fifteen students from the University of California, Berkeley, participated. Their ages ranged from 19 to 30 years. The participants received $\$ 10$ or a class credit for the 1-h experiment.

Apparatus, Stimuli, and Procedure. The apparatus and stimuli were identical to those in Experiment 1. For Experiment 2, only 31 of the previous 37 possible target locations were tested. Targets were never presented at the locations of the landmarks, and the four most lateral positions were not tested.

There were three conditions in Experiment 2. In the presentationonly condition, the landmarks were visible only during the 1-sec presentation phase, together with the target. In the movement-only condition, the landmarks were not present simultaneously with the target but appeared at the offset of the flicker mask. In these conditions, the participants were instructed to ignore the landmarks. In the both-phases condition, the landmarks were present during re- production and movement phases, and the participants were informed that the landmarks remained at the same position.

To familiarize the participants with the general procedure, the experiment started with 5 trials of each condition. The experiment consisted of 18 blocks with 31 trials each, 6 blocks per condition in pseudorandom order. Within each block, the condition remained the same, and the 31 possible target positions were presented in random order. After each block, feedback about average spatial accuracy and MT was given. The participants were encouraged to achieve maximal accuracy while keeping the average MT between 700 and $800 \mathrm{msec}$.

\section{Results and Discussion}

Only $0.5 \%$ of the trials had to be excluded because the movement endpoint missed the target by more than $5 \mathrm{~cm}$. Average RT and MT for the three conditions and for the six target positions immediately surrounding the landmark (near) and the remaining positions (far) can be seen in Table 1. MTs were slightly longer in the both-phases condition than in the presentation-only and movement-only conditions. A repeated measures ANOVA indicated that these differences were marginally significant $[F(2,28)=$ $3.18, p=.057]$.

The pattern of constant error is shown in Figure 4. It is immediately apparent that the distortion patterns in the both-phases and presentation-only conditions were similar to each other and to those shown in Experiment 1 for the both-landmarks condition. In contrast, the responses in the movement-only condition were characterized by a distortion away from the landmarks. To investigate whether these distortion patterns were significant across participants, we used repeated measures ANOVAs on the constant $x$ - and $y$-error within each condition separately. The distortion in the $x$-direction was significant in the presentation-only $[F(3.0,42.8)=6.06, p=.001]$, the movement-only $[F(3.3,46.1)=8.39, p<.001]$, and the bothphases $[F(2.7,37.2)=11.85, p<.001]$ conditions. In the $y$-direction, the distortion was significant in the movementonly condition $[F(1.9,26.3)=12.78, p<.001]$, but not in the presentation-only $[F(1.3,18.8)=2.25, p=.145]$ or the both-phases $[F(1.6,22.2)=3.43, p<.06]$ condition.

An ANOVA comparing the presentation-only and bothphases conditions indicated that the distortion pattern did not differ significantly between these two conditions $[x$, $F(2.7,38.5)=2.15, p=.115 ; y, F(2.2,31.3)=1.09, p=.353]$. In contrast, an ANOVA comparing the movement-only and the both-phases conditions showed a significant interaction between condition and target location $[x, F(3.3,46.0)=$ $8.64, p<.001 ; y, F(3.0,42.2)=20.63, p<.001]$.

The analysis of the constant error indicates that the distortion pattern found in Experiment 1 was not due to the sudden onset of the landmarks at the beginning of the movement phase. Rather, the onset alone, without previous presentation of the landmarks, led to a distortion away from the landmarks. Because the landmarks did not constitute a physical obstacle to the reaching movement, we do not believe that this effect was due to the participants' trying to avoid the landmarks. However, Tipper, Howard, and Houghton (2000) reported that distractors, even if not di- 

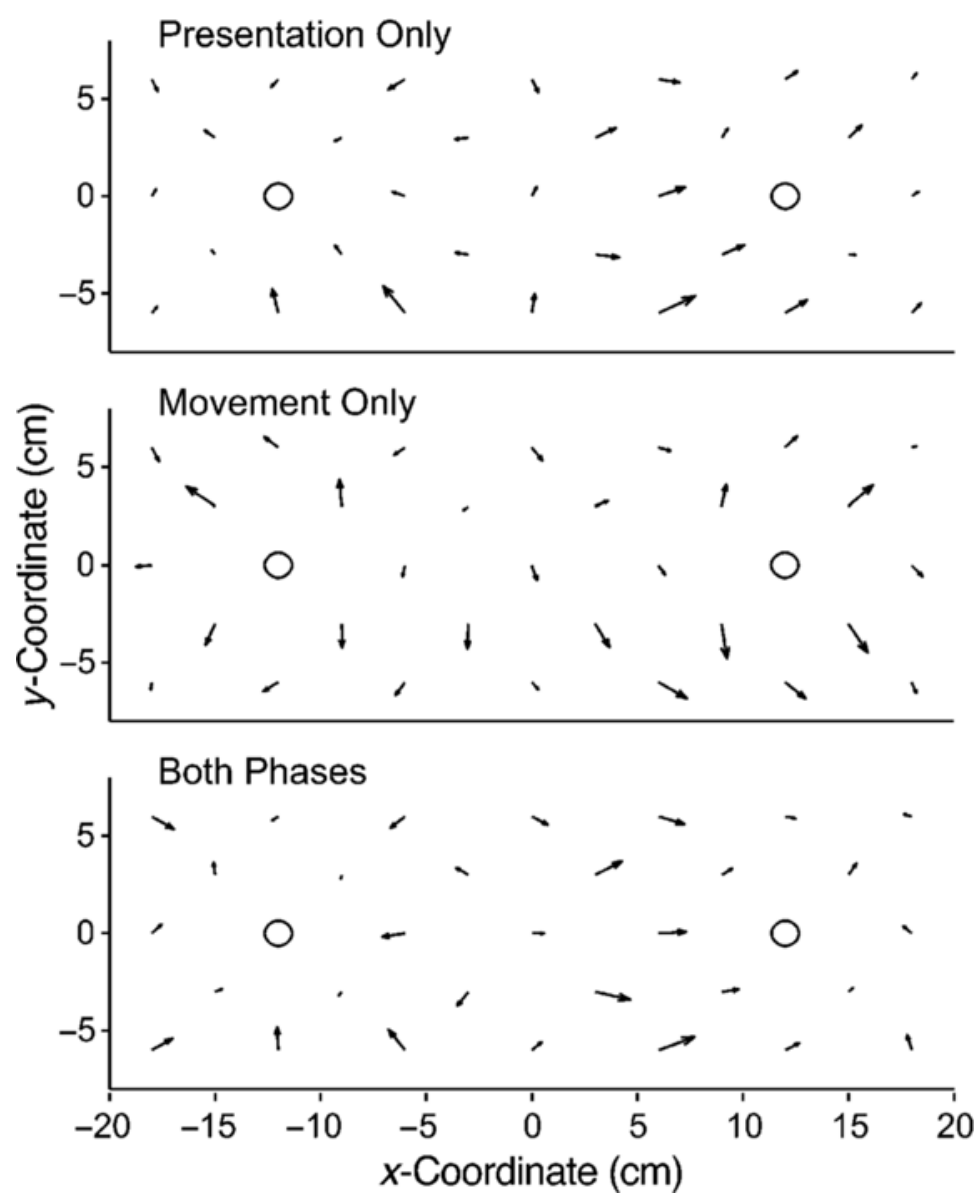

Figure 4. Constant error in Experiment 2 in the presentation-only,movementonly, and both-phases conditions. Errors are magnified threefold.

rectly in the path of the reach, can lead to significant deviation of the hand path away from the distractor location. The authors hypothesized that salient nontarget objects automatically elicit a neural activity in parietal and motor regions that represents a vector in the direction of that location. This activity mixes with the activity related to the reach toward the target. To reach for the target accurately, cells whose preferred direction points toward the distractor have to be suppressed. This would lead to the observed bias away from the distractor location (see also Kerzel, 2002; Suzuki \& Cavanagh, 1997).

The variable error in the three conditions is shown in Figure 5. We analyzed the size of the variable error as the standard deviation of the movement endpoints along the axis of maximal variability (the square root of the largest eigenvalue of the variance/covariance matrix) and separated target positions immediately surrounding a landmark (near) from all the remaining positions (far; see Table 1). A repeated measures ANOVA showed a significant effect of condition $[F(2,28)=17.02, p<.001]$ and a significant effect of target region [far/near; $F(1,14)=6.15, p=.027$ ]. Although the reduction of the variable error in the both-phases condition was especially pronounced near the landmarks
(Table 1), the target region $\times$ condition interaction failed to reach significance $[F(2,28)=3.09, p=.061]$.

Note that the landmarks in the presentation-only condition did not lead to an improvement in accuracy; they were likely ignored in this condition. However, the pattern of constant error was very similar to that observed in the both-phases condition, in which the participants did use the landmarks to improve their accuracy. Thus, nearby distractor objects seemed to have entered the representation of target location for pointing movements in an obligatory fashion, and not only when they actually could be used to improve accuracy. In sum, Experiment 2 provides evidence that the spatial distortions observed in Experiment 1 arose from the common encoding of the target and the surrounding landmarks, rather than from the sudden onset of these stimuli at the beginning of the movement phase.

\section{EXPERIMENT 3}

The previous two experiments indicated that pointing movements are influenced by allocentric informationthat is, the relations between target and landmark locations-after retention intervals as short as $600 \mathrm{msec}$. 

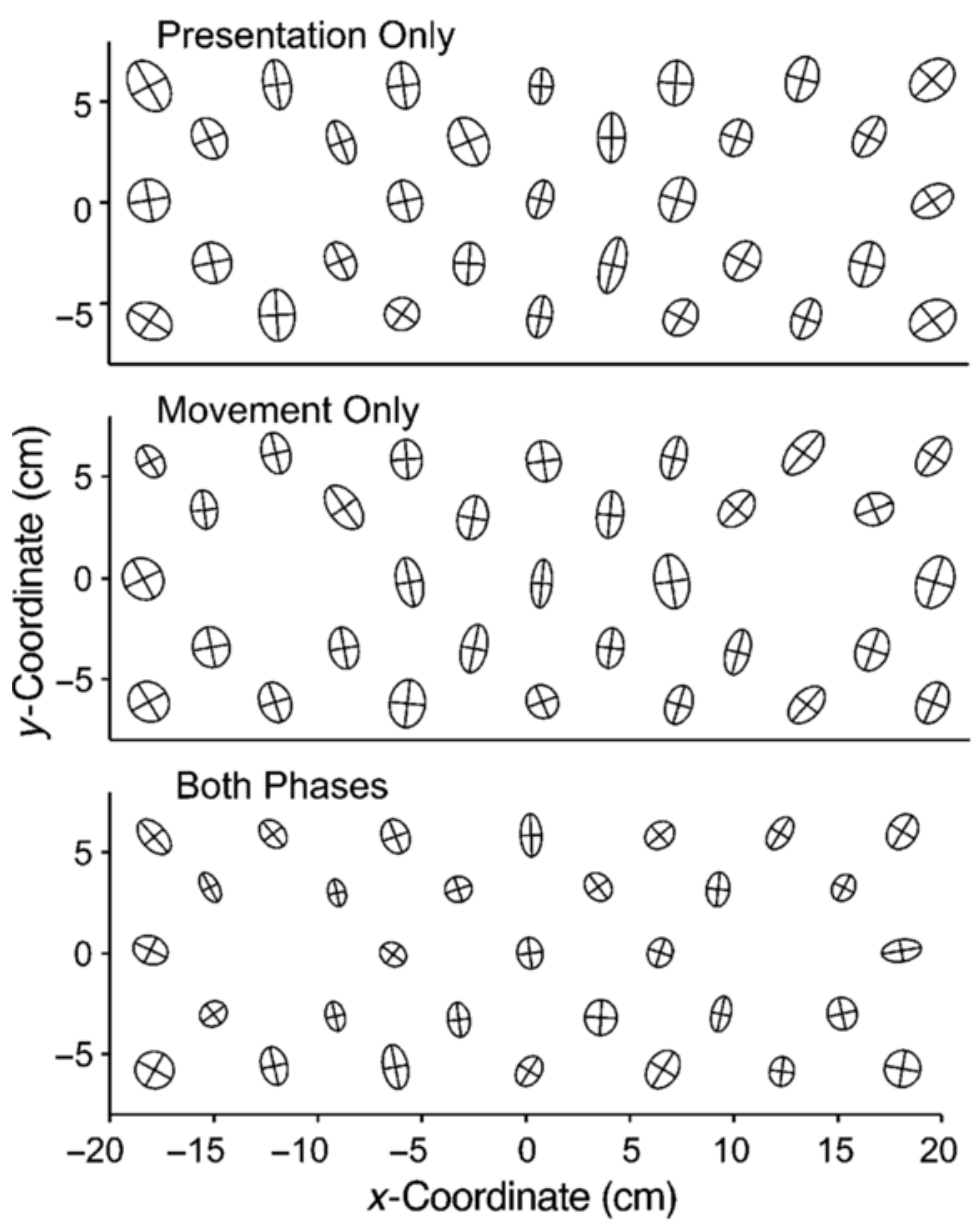

Figure 5. Variable error (1-SD ellipses) in Experiment 2, shown as in Figure 3 , in the presentation-only, movement-only, and both-phases conditions.

However, even such a relatively short delay might lead to a shift from a context-independent egocentric map to a more stable map that includes the relationships of nearby objects (Elliott \& Lee, 1995; Hu \& Goodale, 2000; McIntyre, Stratta, \& Lacquaniti, 1998; Rossetti, 1998).

In Experiment 3, we systematically varied the retention interval in order to investigate whether there would be evidence for an undistorted representation with shorter retention intervals. In addition to delays of 600 and $150 \mathrm{msec}$, we included a condition in which the participants pointed immediately to a target position that was masked for $150 \mathrm{msec}$ only after movement onset. This design allowed us to track the time course of context-dependent distortions. Specifically, we could evaluate the hypothesis that the motor system may rely at first on an undistorted egocentric representation that only later is affected by context-dependent distortion effects.

\section{Method}

Participants. Twelve undergraduates from the University of Göttingen, with ages ranging from 20 to 25 years, were recruited for payment of $15 \mathrm{DM}$ for a single 1-h experimental session.
Apparatus and Stimuli. Experiments 3 and 4 were conducted in a different laboratory, which required a change of apparatus, as compared with Experiments 1 and 2. Responses were performed with a hand-held stylus and recorded with a $45.7 \times 30.5 \mathrm{~cm}$ graphic tablet (Wacom Ultrapad A3, UD-1218). In each trial, the hand started at the lower rim of the sensitive area of the graphic tablet. A movement was considered to have been started when the stylus lost contact with the surface and was considered complete when it touched the surface again. The apparatus allowed full vision of the hand. Stimuli were projected on the surface of the graphic tablet with an LCD projector.

The landmark circles had a diameter of $0.95 \mathrm{~cm}$ and were presented $8.8 \mathrm{~cm}$ to the left and to the right of the sagittal midline. The target dot measured $3.5 \mathrm{~mm}$ in diameter and could be presented at 29 of the possible 65 intersection points of a $13 \times 5$ position grid spanning an area of $26 \times 4 \mathrm{~cm}$ (see the origins of the vectors in Figure 6). The visual mask used in this and the subsequent experiment consisted of a collection of white lines with random positions, lengths, and orientations that changed every $50 \mathrm{msec}$. The lines covered the entire surface of the graphic tablet, rendering $20 \%$ of the surface white in each frame.

Procedure. Three conditions were used in this experiment: immediate reproduction, $150-\mathrm{msec}$ delay, and $600-\mathrm{msec}$ delay. A trial started with the presentation of the two landmarks and the target for $1 \mathrm{sec}$. In the immediate-reproduction condition, a 220-msec-long high-pitched tone served as a starting signal for the pointing movement. As soon as the hand left the starting position, the display was 
Table 2

Reaction Times (RTs), Movement Times (MTs), and

Variable Endpoint Errors (VEs) Along the Axes of Maximal and Minimal Variance in the Three Conditions of Experiment 3

\begin{tabular}{|c|c|c|c|c|c|c|}
\hline \multirow[b]{3}{*}{ Variable } & \multicolumn{6}{|c|}{ Memory Interval } \\
\hline & \multicolumn{2}{|c|}{ Immediate } & \multicolumn{2}{|c|}{$150 \mathrm{msec}$} & \multicolumn{2}{|c|}{$600 \mathrm{msec}$} \\
\hline & Near & Far & Near & Far & Near & Far \\
\hline RT (msec) & 388 & 389 & 253 & 253 & 299 & 297 \\
\hline MT (msec) & 461 & 444 & 491 & 471 & 513 & 496 \\
\hline $\mathrm{VE}$ maximal $(\mathrm{cm})$ & 0.34 & 0.33 & 0.31 & 0.36 & 0.33 & 0.39 \\
\hline VE minimal $(\mathrm{cm})$ & 0.16 & 0.15 & 0.13 & 0.15 & 0.15 & 0.18 \\
\hline
\end{tabular}

Note-Results are shown separately for the six locations immediately surrounding each landmark (near) and for the remaining locations (far).

masked for $150 \mathrm{msec}$ and then presented again without the target while the finger was still in motion. Thus, the landmarks were visible until movement onset and again during the final $80 \%$ of the movement. In the delayed conditions, the visual mask was presented for either 150 or $600 \mathrm{msec}$, and the reappearance of the landmarks served as a starting signal for the pointing movement.

Two practice blocks of 15 trials, one in the $600-\mathrm{msec}$ and one in the immediate-reproduction conditions, were administered at the beginning of the experiment. Each of the three conditions was repeated three times with blocks of 58 trials. The participants alternated between conditions, and the starting condition was counterbalanced across participants.

\section{Results and Discussion}

Trials in which the participants began to move before the starting signal or failed to move at all were excluded from further analysis $(6.8 \%)$. All reproductions deviating more than $5 \mathrm{~cm}$ from the target were also excluded $(0.8 \%)$. The RTs, MTs, and variable errors for locations near the landmark (the 6 positions immediately surrounding each landmark) and far from the landmark (the remaining 17 positions) are shown in Table 2 . RTs were substantially longer in the immediate-reproduction condition; however, the target was still visible in this condition during the RT phase. The effective interval between the disappearance of the target and the start of the pointing movement was $0 \mathrm{msec}$ or slightly negative for the immediate-reproduction condition and, on average, 403 and $898 \mathrm{msec}$ for the 150 - and 600 -msec conditions, respectively. MTs were significantly shortened with decreasing memory intervals $[F(2,22)=$ $6.71, p=.005]$ and were, on average, $18 \mathrm{msec}$ slower when the targets were close to the landmarks $[F(1,11)=131.48$, $p<.001]$; there was no interaction of these variables $[F(2,22)=0.16, p=.85]$.

The constant error (Figure 6) showed an overall pattern similar to that observed in Experiment 1, with distortions away from the midpoint and toward the landmarks. However, in this experiment, we observed a strong global tendency to undershoot the target, likely related to the changed experimental setup. The distortion toward the landmark increased markedly with retention interval, indicated by a strong target position $\times$ retention interval interaction $[x$, $F(6.1,66.6)=6.53, p<.001 ; y, F(3.6,39.3)=4.39, p=$ .006]. Effects of target position on the constant error in the
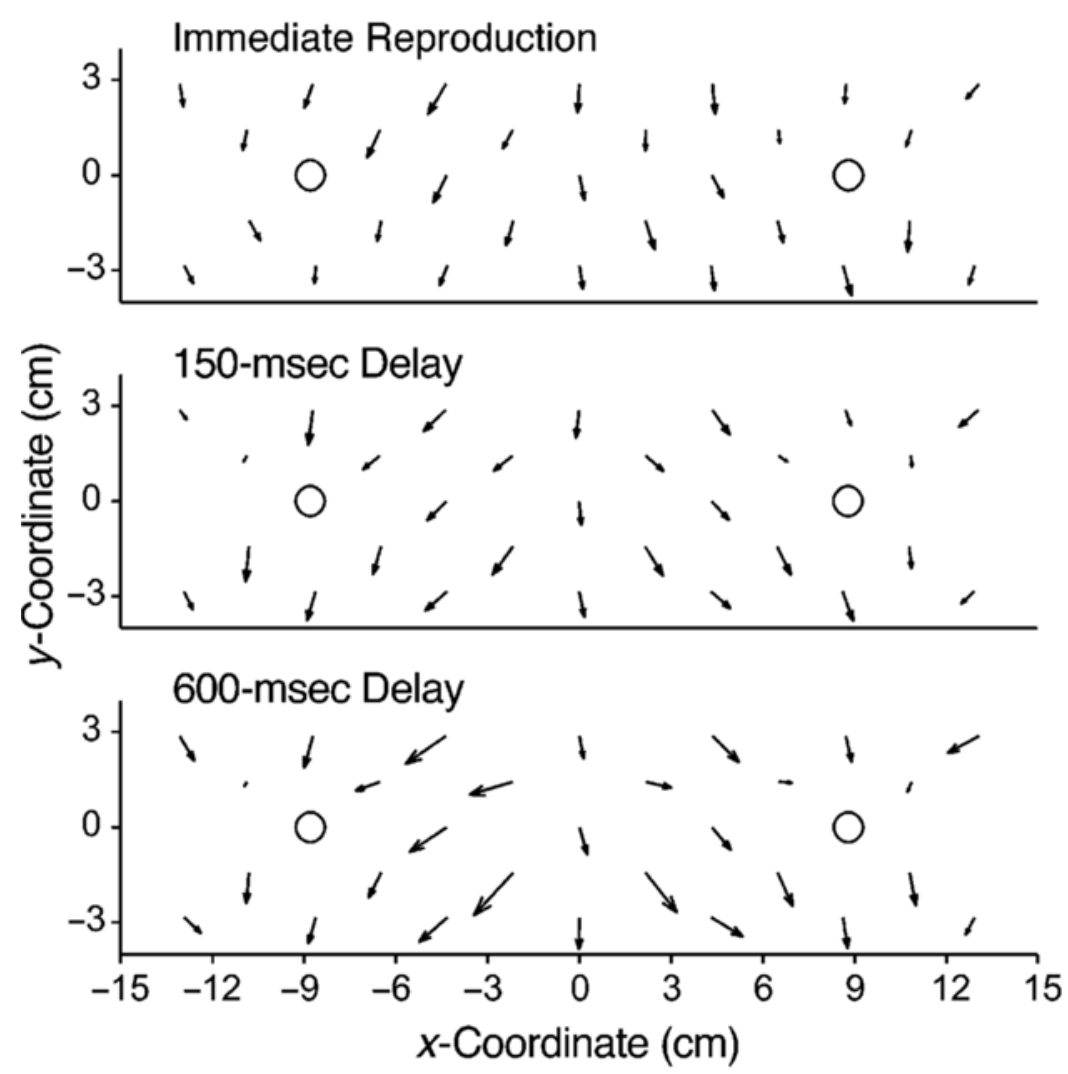

Figure 6. Constant error in Experiment 3. The errors are magnified threefold. 


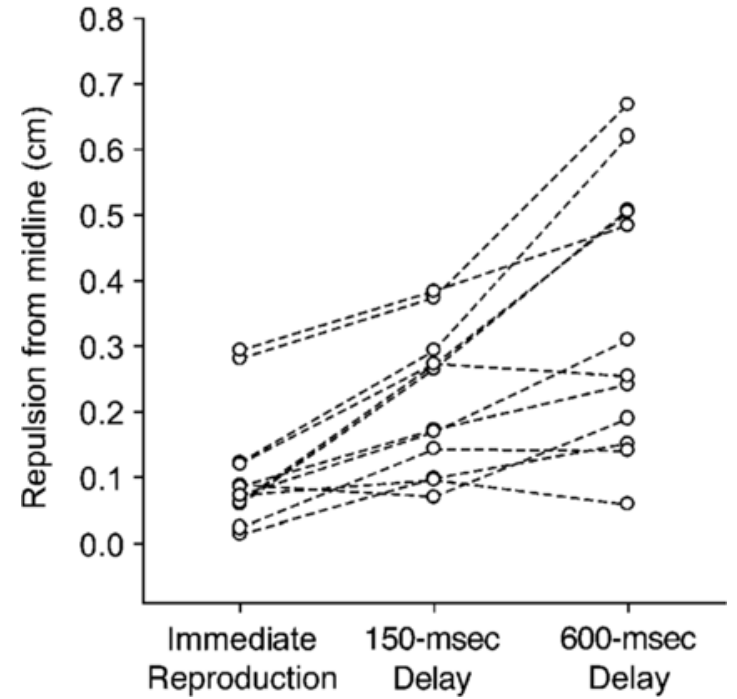

Figure 7. Increase of the distortion in Experiment 3 with increasing memory interval for the 12 individual participants. Shown is the repulsion effect from the midline, averaged across all target positions with a horizontal coordinate of $\pm 4.3 \mathrm{~cm}$.

horizontal direction were significant at each retention interval. This even held true in the immediate-reproduction condition $[F(3.5,38.9)=5.80, p=.001]$, in which the distortion was smaller than but in the same direction as that in the other conditions.

Furthermore, the increase of the distortion, averaged over all the participants, appeared to be smooth. In contrast, Bridgeman et al. (1997) reported that the effect of a visual illusion on pointing responses can be quite abrupt, suggesting a sudden qualitative change from an undistorted to a distorted representation in some participants. To test for this possibility, we quantified the size of each participant's distortions in each condition by measuring the average horizontal repulsion effect from the midline for the target locations with a horizontal coordinate of $\pm 4.3 \mathrm{~cm}$, the locations at which the average pattern showed the largest effect. The average repulsion effect was $0.11 \mathrm{~cm}(S D=0.09)$ in the immediate-reproduction condition, $0.22 \mathrm{~cm}(S D=$ $0.11)$ in the 150 -msec condition, and $0.34 \mathrm{~cm}(S D=0.20)$ in the 600-msec condition (Figure 7). The increase was gradual for all the participants. No participant showed a sudden increase in distortion from one memory interval to the next.

A gradual increase of the influence of the visual context on the remembered location is not unique to pointing movement studies. In earlier experiments, we found a similar pattern for a judgment task. Here, we studied even shorter memory intervals by first presenting the target along with the landmarks, then the visual mask, and finally the landmarks and the target again, but with the target slightly shifted either with or against the direction of expected distortion. The participants' task was to detect the shift. Psychometric functions clearly shifted with retention interval, indicating a gradual build-up of distortion during the first $400 \mathrm{msec}$ of retention. However, distortions were detectable as early as $50 \mathrm{msec}$ after target offset (Diedrichsen, 1998; Werner \& Diedrichsen, 2002).

The variable error (Figure 8, Table 2) also increased with the memory interval. The standard deviation along the axis of maximal variability showed a significant interaction effect between retention interval and distance from the landmark $[F(2,22)=8.19, p=.002]$. The variable error increased for targets far from landmarks but remained constant for targets near landmarks. The variable error along the second axis showed the same significant interaction $[F(2,22)=4.59, p=.022]$. Note that in the immediatereproduction condition, there was no difference in variable error between targets close to landmarks and those far from them.

In summary, these results demonstrate that distortion increases with longer retention intervals, indicating that it is related to changes in the memory representation of the target, not to a general misperception or a motor artifact. The increase was gradual and was not accompanied by qualitative changes in the overall pattern. More specifically, distortions were measurable even if the time between target offset and movement onset was reduced to zero. Variable error also increased for positions far away from the landmarks. Although in the immediate-reproduction condition no difference was found between positions near and far from the landmarks, this difference emerged for longer intervals.

\section{EXPERIMENT 4}

In previous experiments, we have studied the same twolandmarks situation with a perceptual judgment task or a reproduction task with a mouse pointer and have observed a repulsion of the remembered location away from the landmarks (Diedrichsen, 1998; Werner \& Diedrichsen, 2002). It is possible that these differences reflect the fact that participants coded the stimulus positions egocentrically in the pointing task and allocentricallyin the cognitive/perceptual tasks. Alone, context-dependentdistortions, as shown in Experiments $1-3$, do not necessarily imply that the target location was remembered in an allocentric reference frame. Even an egocentric map of space can give rise to contextdependent patterns of distortions when both the target and the landmark are represented within the same map and when the two representations are allowed to interact.

To test whether we would find a distortion effect away from the landmark if the participants were forced to base their pointing movements on allocentric information, we changed the task demands in Experiment 4. The two landmarks were shifted unpredictably during the retention interval, and the participants were instructed to reproduce the target location relative to the landmarks. Therefore, it was not possible to preprogram the motor response; the pointing movement had to be based on the target-to-landmark relation. 


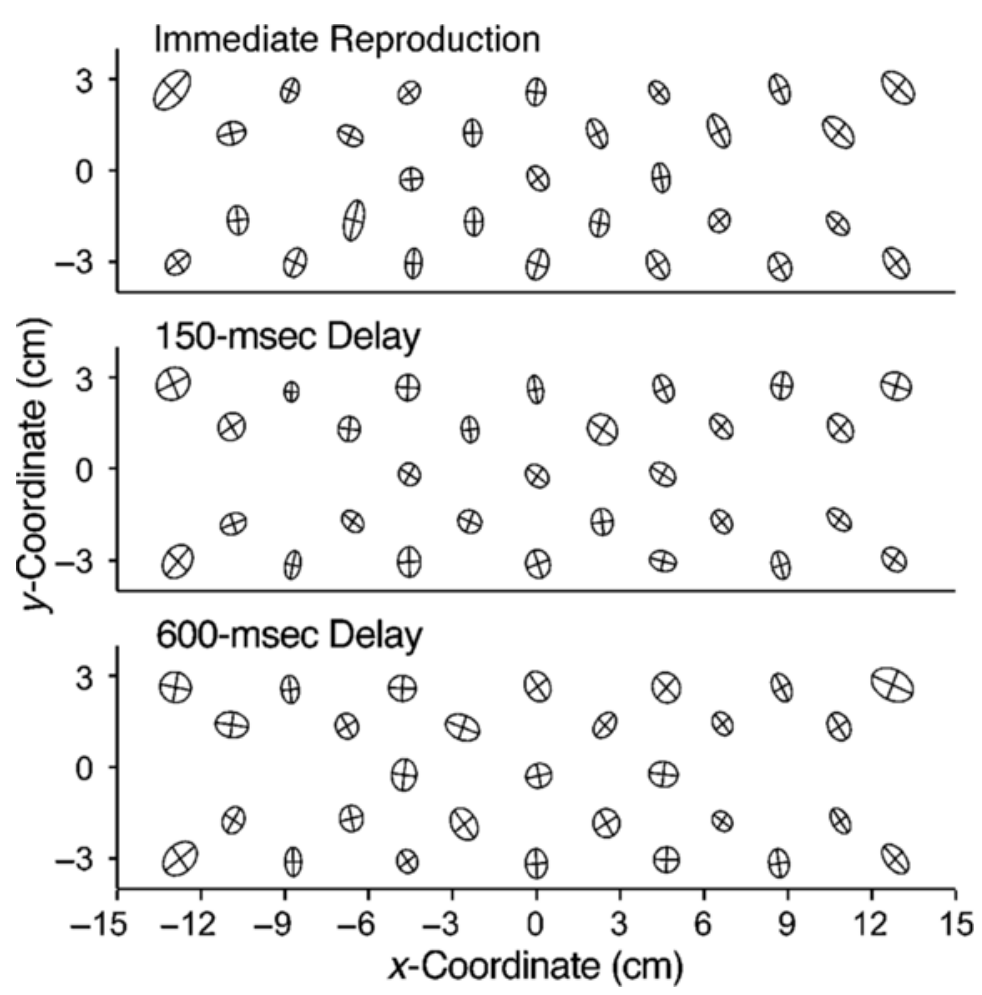

Figure 8. Variable error in Experiment 3 for the immediate-pointing, 150msec delay, and 600-msec delay conditions.

\section{Method}

Participants. Six right-handed participants from the University of Göttingen, Germany, participated for a payment of $15 \mathrm{DM}$ in a single 1-h session.

Apparatus and Stimuli. Experimental setup and stimuli were identical to those in Experiment 3, except that the landmarks were presented $6.5 \mathrm{~cm}$, instead of $8.7 \mathrm{~cm}$, to the left and the right from the midline, to allow for landmark displacements. The possible target locations were scaled accordingly.

Procedure. Each trial began with the presentation of the target along with the two landmarks for $1 \mathrm{sec}$, followed by a masking interval of $600 \mathrm{msec}$. As a starting signal for the pointing movement, the two landmarks appeared again, but shifted by $7.5 \mathrm{~cm}$ relative to their original locations. Shifts occurred unpredictably toward one of the four corners of the graphic tablet. As before, the participants were encouraged to respond quickly. The experiment began with a practice block of 20 trials, followed by three experimental blocks of 116 trials.

\section{Results and Discussion}

Trials with movements starting before mask offset (6.9\%) or with MTs longer than $700 \mathrm{msec}(2.3 \%)$ were excluded from data analysis. Responses that deviated from the target by more than $5 \mathrm{~cm}$ were also excluded $(0.8 \%)$. The average RT was $354 \mathrm{msec}(S D=45 \mathrm{msec})$, and the average MT was $505 \mathrm{msec}(S D=66 \mathrm{msec})$.

The overall pattern of constant error (Figure 9, upper panel) was significant across participants $[x, F(3.2,15.8)=$ $14.43, p<.001 ; y, F(2.1,10.4)=15.64, p=.001]$. Importantly, we did not find a repulsion effect away from the landmarks, as has been observed with other experimental tasks (Werner \& Diedrichsen, 2002). Although the pattern was much more pronounced than that in each of Experiments $1-3$, a qualitatively similar pattern with marked distortion away from the midpoint and toward the landmarks was found. Correlating each individual's distortion pattern in this experiment (both $x$ - and $y$-components) with each individual's pattern in the 600-msec condition of Experiment 3 $(6 \times 12=72$ possible pairings $)$ yielded an average correlation of .66 .

The variable error (Figure 9, lower panel) was clearly reduced for the six positions immediately surrounding each landmark, as compared with the seven positions near the midpoint between the landmarks $[0.34 \mathrm{vs} .0 .55 \mathrm{~cm}$ for the maximal component, $F(1,5)=191.6, p<.001 ; 0.20$ vs. $0.27 \mathrm{~cm}$ for the minimal component, $F(1,5)=37.94, p=.002]$. The variable error in this experiment was substantially larger than that in the previous experiment, showing that egocentric information was used to improve accuracy in Experiments $1-3$. The axis of maximal variability was directed toward the landmarks, whereas in the no-landmark condition of Experiment 1, these were aligned toward the person. This reflects the two reference frames required by the different tasks (Carrozzo et al., 2002; Rossetti, 1998). The pattern of variable error when the landmarks did not shift appears to be a mixture of these two patterns, indicating that both egocentric and allocentric information was used. 


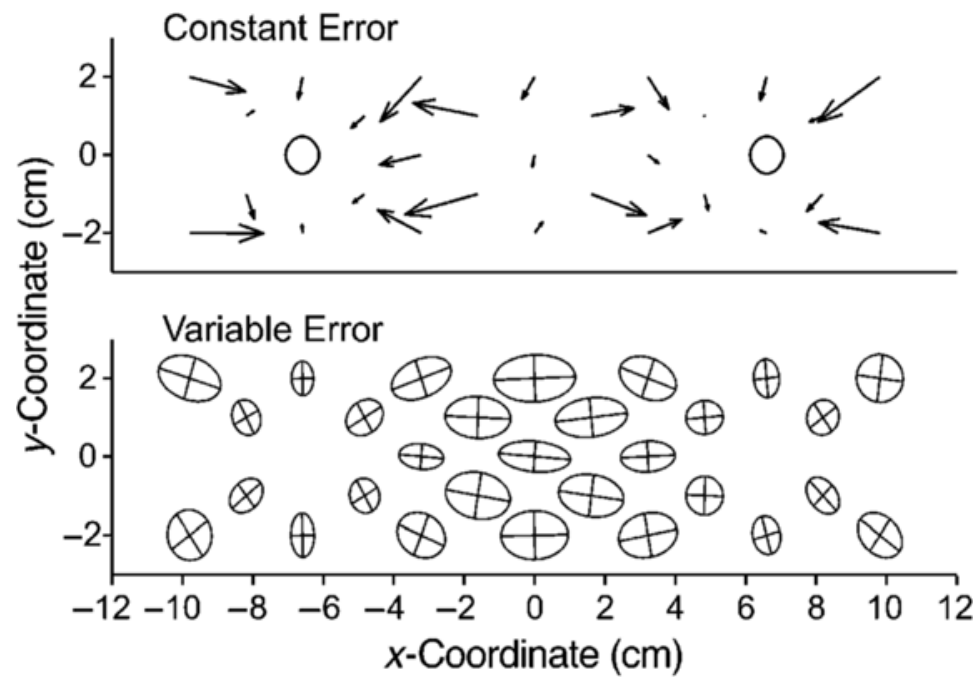

Figure 9. Constant and variable error in Experiment 4. Constant error is shown magnified by a factor of two (the other figures used a larger magnification of three). The ellipses indicate $1 S D$ dispersion of movement endpoints around the individual's average reproduced location.

In this experiment, it was not possible to memorize the position of the target exclusively in egocentric coordinatesfor example, as a preplanned motor program. The spatial relations between the target and the landmarks had to be encoded and used to guide the movement. The results suggest that the distortion arising in this situation does not differ qualitatively from the distortion patterns in the previous experiments. This adds credibility to our assertion that distortion stems from coding of the spatial relation between target and landmarks.

\section{GENERAL DISCUSSION}

In this article, we investigated the short-term memory representation underlying pointing movements to memorized targets. Specifically, the question was whether an early memory representation that is egocentrically organized and immune to context-dependentspatial distortions can be utilized to guide movements (Bridgeman et al., 1997; Hu \& Goodale, 2000; Milner \& Goodale, 1995). Experiment 1 showed that visual nontarget landmarks had a systematic influence on the endpoints of the pointing movements, which is evident both in decreased positional uncertainty near the landmarks and in the pattern of constant error away from the midpoint and toward the landmarks. These effects were clearly stimulus centered, as was shown by the differential effects of displays containing only the left, only the right, both, or no landmarks.

In other studies, we have investigated distortions in visual memory for targets in the same constellation of landmarks, using reproductions of the position with a mouse cursor or same/different judgments as response modalities (Diedrichsen, 1998; Werner \& Diedrichsen, 2002; Werner \& Schmidt, 2000). Unlike in the experiments re- ported here, the landmarks induced a repulsion effect on the memorized locations for targets in close proximity to the landmarks.

We do not believe that these differences in the patterns of constant error are due to a fundamental difference in the spatial representations used for these tasks. One may argue that pointing movements are based on egocentric representations, whereas judgments are based on allocentric representations. However, in Experiment 4, we did not find any repulsion effect away from the landmarks, even when the participants were forced to base their pointing movements on allocentric information.

We hypothesize that, rather than reflecting qualitatively different systems for the two response modalities, differences between the experiments may be due to eye movements or attentional factors. Sheth and Shimojo (2001) recently demonstrated that memory of spatial location is biased toward fixation and toward salient landmarks and that this distortion increases with longer memory intervals. In contrast, a repellent effect of attended, but nonfixated, locations has been reported (Kerzel, 2002; Suzuki \& Cavanagh, 1997). When pointing with the mouse, participants may spend more time fixating the cursor than they do during natural pointing movements. However, in our study, the length of the encoding phase allowed several exploratory saccades. We neither restricted nor recorded eye movements, which limits the conclusions that can be made from this study about their role in producing the distortion effects.

In Experiment 2, we tested whether distortion might be due to the sudden onset of the landmarks after the memory interval. When the landmarks were presented only in the movement phase, a qualitatively different pattern was found, characterized by a distortion away from the land- 
marks. The participants reported having focused on the remembered target position, so the repellent effect of the landmark is likely related to the repellent effect reported for nonfixated irrelevant distractors (Kerzel, 2002; Suzuki \& Cavanagh, 1997). In contrast, the distortion observed in the presentation-only condition was similar to the distortion observed when the landmarks were present in both phases, characterized by a distortion toward the landmark. This indicates that the influence of landmarks on short-term memory is not limited to situations in which they improve accuracy.

Experiment 3 showed that the pattern of spatial distortions is already present when pointing movements are initiated under full vision of the target. We also found that the distortion increased in magnitude when the retention interval was lengthened to 150 and $600 \mathrm{msec}$. It has been proposed that immediate actions are based on a contextindependent egocentric map that can stay unaffected by visual illusions for as long as 2-4 sec (Bridgeman et al., 1997). When the action is delayed further, that representation deteriorates, and the action become increasingly influenced by visual context (Bridgeman et al., 1997; Gentilucci et al., 1996; Hu \& Goodale, 2000). Although our results confirm the time dependency of context-dependent effects, they are incongruent with the observation that the participants switched from an undistorted toward a distorted representation in an all-or-none fashion (Bridgeman et al., 1997). Rather, the distortion developed gradually over time and had a very early onset. One critical difference between our study and the results reported by Bridgeman et al. (1997) could be the fact that, in the latter study, the display was not masked after target presentation, making it possible for the participants to rely on the visual afterimage of the target to guide the pointing movement. This strategy would have been feasible only for a limited time (in darkness, probably in the order of multiple seconds), after which the participants would have had to rely on a distorted memory representation.

Most important, we observed a significant influence of the landmarks on the pointing movement even when there was no delay between target offset and start of the movement. We were able to map the emergence of contextdependent effects on pointing movements for much shorter retention intervals than had previously been done (Bridgeman et al., 1997; Gentilucci et al., 1996; Hu \& Goodale, 2000). Furthermore, our results extended previous findings by Gourtzelidis et al. (2001) and showed that the early distortion is present even under natural pointing conditions. The early presence of these effects clearly contradicts the hypothesis that a pure context-independent egocentric representation can be used to guide an action. In Experiment 2, we provided evidence that the landmarks introduced a distortion, although they could not be used strategically to improve accuracy.

There are two possible ways to account for these data. In a two-representation framework, a gradual shift can be accounted for if one proposes that pointing movements are based on a mixture between a context-independent ego- centric map and a map that includes the relative position of the objects with respect to one another. The accurate egocentric representation decays rapidly, leading to an increasing influence of the context-dependent representation. Although our results are not inconsistent with this framework, they indicate that a context-independent egocentric representation, should it indeed exist, is immediately accompanied by a context-dependentrepresentation and that the weights of these two representations change smoothly over time. A sudden switch between representations or strategies is not compatible with our results.

Alternatively, a model that assumes one unitary underlying representation can also account for the data. As was pointed out above, the presence of stimulus-dependent visual distortions does not imply an allocentric reference frame. Instead, locations for pointing movements might be represented in a map coded relative to an egocentric reference frame but including multiple objects and their interrelations. As information on the target location begins to decay (Gnadt, Bracewell, \& Andersen, 1991; White, Sparks, $\&$ Stanford, 1994), the influence of the other encoded stimuli in this map increases. Indeed, neural network simulations demonstrate that an increase of lateral interactions of targets and nontargets can lead to distortion patterns similar to the ones reported here (Trommershäuser, 2001).

The latter view would imply that the dorsal stream represents the target of the upcoming action together with other objects in the surrounding context in the same representational medium. Such a view is consistent with the observation that multiple spatial locations can be represented simultaneously in dorsal motor areas (Cisek \& Kalaska, 2002). It would also account for the difficulty of showing a dissociation between the sensorimotor and the cognitive modes in terms of susceptibility to visual illusions (Bruno, 2001; Franz, 2001). A representation of multiple objects in the dorsal stream likely has functional benefitsfor example, for the avoidance of obstacles (Sabes \& Jordan, 1997).

\section{REFERENCES}

Bridgeman, B., Gemmer, A., Forsman, T., \& Huemer, V. (2000). Processing spatial information in the sensorimotor branch of the visual system. Vision Research, 40, 3539-3552.

Bridgeman, B., LeWIS, S., Heit, G., \& NAGLE, M. (1979). Relation between cognitive and motor-oriented systems of visual position perception. Journal of Experimental Psychology: Human Perception \& Performance, 5, 692-700.

Bridgeman, B., PEERY, S., \& ANANd, S. (1997). Interaction of cognitive and sensorimotor maps of visual space. Perception \& Psychophysics, 59, 456-469.

BRUNO, N. (2001). When does action resist visual illusions? Trends in Cognitive Sciences, 5, 379-382.

Buneo, C. A., Jarvis, M. R., Batista, A. P., \& Andersen, R. A. (2002). Direct visuomotor transformations for reaching. Nature, 416, 632636.

Carrozzo, M., Stratta, F., Mcintyre, J., \& Lacquaniti, F. (2002). Cognitive allocentric representations of visual space shape pointing errors. Experimental Brain Research, 147, 426-436.

Cise K, P., \& Kalaska, J. F. (2002). Simultaneous encoding of multiple potential reach directions in dorsal premotor cortex. Journal of $\mathrm{Neu}$ rophysiology, 87, 1149-1154. 
DIEDRICHSEN, J. (1998). Gibt es ein motorisches Kurzzeitgedächtnis für Positionen im Greifraum? Unpublished diploma thesis, University of Göttingen.

Elliott, D., \& LeE, T. D. (1995). The role of target information on manual-aiming bias. Psychological Research, 58, 2-9.

Flanders, M., Tillery, S. I. H., \& Soechting, J. F. (1992). Early stages in a sensorimotor transformation. Behavioral \& Brain Sciences, 15, 309-362.

Franz, V. H. (2001). Action does not resist visual illusions. Trends in Cognitive Sciences, 5, 457-459.

Funahashi, S., Bruce, C. J., \& Goldman-Rakic, P. S. (1989). Mnemonic coding of visual space in the monkey's dorsolateral prefrontal cortex. Journal of Neurophysiology, 61, 331-349.

Gentilucci, M., Chieffi, S., Daprati, E., Saetti, M. C., \& Toni, I. (1996). Visual illusion and action. Neuropsychologica, 34, 369-376.

GNADT, J. W., \& ANDERSEN, R. A. (1992). Memory related motor planning activity in posterior parietal cortex of macaque. In S. M. Kosslyn $\&$ R. A. Andersen (Eds.), Frontiers in cognitive neuroscience (pp. 468 472). Cambridge, MA: MIT Press.

Gnadt, J. W., Bracewell, R. M., \& Andersen, R. A. (1991). Sensorimotor transformation during eye movements to remembered visual targets. Vision Research, 31, 693-715.

GOLDMAN-RAKIC, P. S. (1995). Cellular basis of working memory. Neuron, 14, 477-485.

Goodale, M. A., Milner, A. D., Jakobson, L. S., \& Carey, D. P. (1991). A neurological dissociation between perceiving objects and grasping them. Nature, 349, 154-156.

GourTZELIDIS, P., SMYRNis, N., Evdokimidis, I., \& BALOGH, A. (2001). Systematic errors of planar arm movements provide evidence for space categorization effects and interaction of multiple frames of reference. Experimental Brain Research, 139, 59-69.

Hu, Y., \& Goodale, M. A. (2000). Grasping after a delay shifts sizescaling from absolute to relative metrics. Journal of Cognitive Neuroscience, 12, 856-868.

Huttenlocher, J., Hedges, L. V., \& Duncan, S. (1991). Categories and particulars: Prototype effects in estimating spatial location. Psychological Review, 98, 352-376.

KeLso, J. A. (1977). Motor control mechanisms underlying human movement production. Journal of Experimental Psychology: Human Perception \& Performance, 3, 529-543.

KERZEL, D. (2002). Memory for the position of stationary objects: Disentangling foveal bias and memory averaging. Vision Research, 42, 159-167.

LAABS, G. J. (1973). Retention characteristics of different reproduction cues in motor short-term memory. Journal of Experimental Psychology, 100, 168-177.

Mcintyre, J., Stratta, F., Droulez, J., \& Lacquaniti, F. (2000). Analysis of pointing errors reveals properties of data representations and coordinate transformations within the central nervous system. Neural Computation, 12, 2823-2855.

MCINTYRe, J., StRATta, F., \& LACQUANiti, F. (1998). Short-term memory for reaching to visual targets: Psychophysical evidence for bodycentered reference frames. Journal of Neuroscience, 18, 8423-8435.

Merigan, W. H., \& MAUnSELl, J. H. (1993). How parallel are the primate visual pathways? Annual Review of Neuroscience, 16, 369-402.

Milner, A. D., \& Goodale, M. A. (1995). The visual brain in action. Oxford: Oxford University Press.

Milner, A. D., Perret, D. I., Johnston, R. S., Benson, P. J., Jordan, T. R., Heeley, D. W., Bettucci, D., Mortara, F., Mutani, R., TerRAZZI, E., \& DAVIDSON, D. L. W. (1991). Perception and action in "visual form agnosia." Brain, 114, 405-428.

NEGGERS, S. F., \& BEKKERING, H. (2000). Ocular gaze is anchored to the target of an ongoing pointing movement. Journal of Neurophysiology, 83, 639-651.

Nelson, T. O., \& Chaiklin, S. (1980). Immediate memory for spatial location. Journal of Experimental Psychology: Human Learning \& Memory, 6, 529-545.

Pelisson, D., Prablanc, C., Goodale, M. A., \& Jeannerod, M. (1986). Visual control of reaching movements without vision of the limb: II. Evidence of fast unconscious processes correcting the trajectory of the hand to the final position of a double-step stimulus. Experimental Brain Research, 62, 303-311.

Perenin, M. T., \& Vighetto, A. (1988). Optic ataxia: A specific disruption in visuomotor mechanisms: I. Different aspects of the deficit in reaching for objects. Brain, 111, 643-674.

Pouget, A., \& SEJNOwSKI, T. (1997). Spatial transformations in the parietal cortex using basis functions. Journal of Cognitive Neuroscience, 9, 222-237.

RosSETTI, Y. (1998). Implicit short-lived motor representations of space in brain damaged and healthy subjects. Consciousness \& Cognition, 7, 520-558.

SABES, P. N., \& JORDAN, M. I. (1997). Obstacle avoidance and a perturbation sensitivity model for motor planning. Journal of Neuroscience, 17, 7119-7128

SCHMidT, T., Werner, S., \& DiedrichSEn, J. (2003). Spatial distortions induced by multiple visual landmarks: How local distortions combine to produce complex distortion patterns. Perception \& Psychophysics, 65, 861-873.

Sheth, B. R., \& Shimojo, S. (2001). Compression of space in visual memory. Vision Research, 41, 329-341.

Smyrnis, N., Taira, M., Ashe, J., \& Georgopoulos, A. P. (1992). Motor cortical activity in a memorized delay task. Experimental Brain Research, 92, 139-151.

Stelmach, G. E., Kelso, J. A., \& Wallace, S. A. (1975). Preselection in short-term motor memory. Journal of Experimental Psychology: Human Perception \& Performance, 1, 745-755.

SuzuKi, S., \& CAVANAGH, P. (1997). Focused attention distorts visual space: An attentional repulsion effect. Journal of Experimental Psychology: Human Perception \& Performance, 23, 443-463.

Tipper, S. P., Howard, L. A., \& Houghton, G. (2000). Behavioral consequences of selection from neural population codes. In S. Monsell \& J. Driver (Eds.), Control of cognitive processes: Attention and performance XVIII (pp. 223-245). Cambridge, MA: MIT Press.

TROMMERSHÄUSER, J. (2001). Strategieabhaengige Verzerrungen im visuell-räumlichen Arbeitsgedaechtnis. Unpublished diploma thesis, University of Göttingen.

WERNER, S., \& DIEDRICHSEN, J. (2002). The time course of spatial memory distortions. Memory \& Cognition, 30, 718-730.

WERNER, S., \& SCHMIDT, T. (2000). Investigating spatial reference systems through distortions in visual memory. In C. Freska, C. H. Brauer, $\&$ K. F. Wender (Eds.), Spatial cognition II: Integrating abstract theories, empirical studies, formal methods, and practical applications (pp. 169-183). Berlin: Springer-Verlag.

White, J. M., SPARKS, D. L., \& STANFord, T. R. (1994). Saccades to remembered target locations: An analysis of systematic and variable errors. Vision Research, 34, 79-92.

YANTIS, S., \& JONIDES, J. (1990). Abrupt visual onsets and selective attention: Voluntary versus automatic allocation. Journal of Experimental Psychology: Human Perception \& Performance, 16, 121-134.

\section{NOTES}

1. In this article, we will use the term spatial distortion as indicating the pattern of constant errors (the average distance between reproduced and actual target positions) across the tested field.

2. We thank Dan Simons for raising this possibility

(Manuscript received August 16, 2002; revision accepted for publication June 3, 2003.) 